\title{
Comparison of Accuracy Among Pedometers from Five Japanese Manufacturers
}

\author{
So Osawa ${ }^{1,{ }^{*}}$, Hisaaki Tabuchi ${ }^{1}$, Kenichi Nemoto ${ }^{2}$, Shuhei Tokimasa ${ }^{1}$, Shotaro Misaki ${ }^{1}$, Masao \\ Okuhara $^{3}$ and Koji Terasawa ${ }^{4}$
}

\author{
${ }^{1}$ Shinshu University, Faculty of Education, ${ }^{2}$ Human Science, Matsumoto University ${ }^{3}$ Management Information, Tokyo \\ University of Science, Suwa, ${ }^{4}$ Shinshu University Graduate School of Education and Medicine, Japan
}

\begin{abstract}
The reliability of commercially available pedometers is not known in detail. Therefore, the present study examines the accuracy and reliability of the FS500 (Acos), HJ301 (Omron), EX700 (Yamax), FB727 (Tanita) and TW600 (Citizen) pedometers to count steps and measure energy expenditure at various walking speeds. Twenty individuals (age, $32.5 \pm 15.3$ years; body mass index, $22.0 \pm 1.6 \mathrm{~kg} / \mathrm{m}^{2}$ ) walked at three speeds for $6 \mathrm{~min}$. Step-counts and energy expenditure determined by each pedometer were compared with actual values. All five pedometers accurately measured steps at all speeds, but tended to underestimate expended calories to within $50 \%$ of the actual amount of energy expenditure. The correlation coefficients $(R)$ between actual energy expenditure and pedometer values were between 0.74 and 0.87 . Thus, feedback about energy expenditure is somewhat inaccurate. In contrast, step counts are very accurate, and thus pedometers are useful tools with which to indicate daily exercise levels.
\end{abstract}

Keywords: Energy Expenditure, Kilocalories, Pedometer, Speed, Step Counter, Walking.

\section{INTRODUCTION}

Health promotion has become a global effort since the Ottawa WHO Charter of 1989 [1] and based on this charter, the "Kenko Nippon 21" program formulated in 2000 aimed to reduce mortality rates among late-middle-aged individuals, prolong healthy lives, improve quality of life and promote regional health [2]. Kenko Nippon 21 states that a decrease in physical activity is not only a risk factor for obesity and lifestyle diseases, but also for chronic disease or infirmity among elderly individuals. Physical activity can be determined as the number of steps taken per day. The 1997 counts of 8,202 and 7,282 steps per day for males and females in Japan, respectively, resulted in Kenko Nippon 21 establishing a target of 9,200 and 8,300 steps, respectively. However, the number of actual steps significantly decreased to 7,243 and 6,431 for men and women, respectively. The numbers of steps taken daily by men and women aged over 70 years in 1997 were 5,436 and 4,604, respectively, and targets were set at 6,700 and 5,900, respectively. These had also significantly decreased to 4,707 and 3,917 respectively, by 2010 .

The benefits of exercise have become more obvious, and exercise programs can effectively improve health and fitness. The American College of Sports Medicine recommends at least 30 min of physical activity at moderate intensity on most days of the week [3]. Increasing the amount of physical activity leads to a lower incidence of lifestyle-related

*Address correspondence to this author at the Shinshu University, Faculty of Education 6-Ro Nishi-Nagano, Nagano 380-8544, Japan;

Tel: 026-238-4213; Fax: 026-238-4213; E-mail: 12ea502e@shinshu-u.ac.jp diseases [4]. Hatano suggested that walking 10,000 steps/day would effectively prevent cardiovascular disease [5]. Moreover, Paffenbargher reported that expending more than 2000 kilocalories (kcal) per week would reduce the risk of heart attack [6]. Walking 10,000 steps/day improves glucose tolerance and reduces systolic blood pressure, which are risk factors for type 2 diabetes [7, 8]. Epidemiological studies also support the notion that physical activity can lower the risk of coronary artery disease, hypertension, diabetes and obesity [9-12].

Electronic pedometers can help to increase daily physical activity levels. A few studies have shown that individuals walk further and for longer periods with than without pedometers [10,13-15]. Daily physical activity can now be measured using inexpensive, practical electronic pedometers. Giannakidoku et al. [16] stated that most of today's electronic pedometers measure numbers of steps and expended energy, but these values vary. The findings of several studies suggest that most pedometers accurately measure numbers of steps at walking speeds of fast speeds $(\geq 67-80 \mathrm{~m} / \mathrm{min})$ and become even more accurate with increasing walking speed, but tend to underestimate steps at walking speeds of slow speeds $(\leq 67-80 \mathrm{~m} / \mathrm{min})$ and become even more inaccurate with decreasing walking speed [1623]. According to Japanese Industrial Standards (JIS) regulations set by the Ministry of Industrial and Trading, the maximum permissible rate of miscounted steps is 3\% [5]. Some authors have recommended Japanese pedometers for daily use $[18,24]$. However most pedometers do not show accurate energy expenditure in kilocalories and some research points out the difficulty in measuring energy expenditure using currently distributed pedometers. The 
Table 1. Characteristics of Participants

\begin{tabular}{|c|c|}
\hline & All (N=20) \\
\hline \hline Height $(\mathrm{cm})$ & $166.1 \pm 5.1$ \\
\hline Weight $(\mathrm{kg})$ & $60.5 \pm 4.8$ \\
\hline Age $(\mathrm{y})$ & $32.5 \pm 15.3$ \\
\hline Stride $(\mathrm{cm} / \mathrm{step})$ & $74.8 \pm 2.2$ \\
\hline Body mass index $\left(\mathrm{kg} / \mathrm{m}^{2}\right)$ & $22.0 \pm 1.6$ \\
\hline
\end{tabular}

Values are shown as means $\pm \mathrm{SD}$

OMRON HJ-720 and HJ-113 pedometers speed-dependently underestimate energy expenditure measured as kilocalories by up to $28 \%$ [16]. Conversely, the OMRON HJ-700IT pedometer tends to overestimate energy expenditure at various speeds [25]. Crouter et al. [18] found that eight pedometers generated higher-than-average amounts of expended calories compared with actual values. They questioned whether pedometers in fact measure gross or net kilocalories. Valid measurement devices are important to determine the appropriate device with which to analyze physical activity. Therefore, the present study examines the accuracy and reliability of counting steps and measuring energy expenditure at various walking speeds using electronic pedometers from five Japanese manufacturers.

\section{METHODS}

\section{Participants}

Twenty untrained volunteers (16 men and 4 women) volunteered to participate in this study. This study complied with the human subject policy of Shinshu University. All participants were informed of the potential experimental risks, and they gave their written informed consent for their participation in this study. Height was measured without shoes using a stadiometer and weight was also assessed without shoes in light clothing using a calibrated physician's scale. In addition, each subject's stride length was determined before participation in the study. To determine stride length, subjects were asked to take 20 strides at their normal walking speed on a flat floor in a gym. The total distance was divided by 20 , to calculate average stride length. Table 1 shows the physical characteristics of the participants.

\section{Protocol}

The participants wore all five pedometers while walking at $100(\mathrm{P} 100 ; 75 \mathrm{~m} / \mathrm{min}), 120(\mathrm{P} 120 ; 90 \mathrm{~m} / \mathrm{min})$ and 140 (P140; $105 \mathrm{~m} / \mathrm{min}$ ) steps per minute for $6 \mathrm{~min}$ at $10-\mathrm{min}$ intervals. The participants timed their steps to a metronome at each speed before beginning the trials to accurately total the numbers of actual steps. The five pedometers simultaneously recorded and displayed step counts and energy expenditure. A researcher hand-tallied the actual numbers of steps taken while walking behind each participant to avoid influencing their walking speed. Actual energy expenditure was determined using a METAVINE VMB-005N (VINE Ltd., Tokyo, Japan) system that measures metabolism. The participants wore a 21900BZX01223000 facemask (TOKIBO Co. Ltd., Tokyo, Japan) that measured energy expenditure. The participants walked on a flat gym floor for $50 \mathrm{~m}$ while wearing the pedometers around the waist.

Step counts and energy expenditure recorded by the five pedometers (pedometer values) were compared with the actual values for numbers of steps and energy expenditure.

\section{Pedometers}

We assessed the following commercially available, electronic pedometers that are popular in Japan: FS500 (Acos Co. Ltd., Iida, Japan; AC), HJ301 (Omron Corporation, Kyoto, Japan; OM), Yamax EX700 (Yamasa Tokei Keiki Co. Ltd., Tokyo, Japan; YAMX), FB727 (Tanita Corporation, Tokyo, Japan; TNT), and TW600 (Citizen Systems Japan Co., Ltd., Tokyo, Japan; CTZ).

\section{Statistical Analysis}

The correlation coefficient termed $R$ was calculated between actual expended calories and pedometer values. A two-way repeated measured ANOVA was used to assess differences between the pedometer speed and to determine whether significant differences existed between pedometer models. Paired t-tests were used to determine whether the pedometer-estimated steps and calories were significantly different from the actual steps and calories taken. The level of significance was set at $p<0.05$.

Statistical analyses were performed using SPSS 11.0.1 Statistical Packages (SPSS Inc., Chicago, USA). The significance level was set at 0.05 .

\section{RESULTS}

\section{Accuracy of Step Counts}

All pedometers accurately counted steps at all speeds, as all values were within $\pm 3 \%$ of the actual counts $(F=1.7, p>$ $0.05)$. Fig. (1) shows the ratios (\%) of pedometer values based on the actual number of steps at each walking speed and Table 2 shows the statistical results of the actual steps as follows: P100: AC, 100.9\% ( $\mathrm{P}<0.05)$; OM, 100.9\% $(\mathrm{P}<$ $0.01)$; YAMX, 101.8\%; TNT, 100.5\%; CTZ, 101.8\% (P< 0.001); P120: AC, 100.7\%; OM, 100.0\%; YAMX, 100.5\%; TNT, 99.5\%; CTZ, 100.9\% (P < 0.01); P140, AC, 99.6\%; OM, 99.5\%; YAMX, 99.6\%; TNT, 97.2\% (P < 0.05); CTZ, $99.6 \%$. The differences between actual and all pedometercounted steps were within $\pm 2 \%$ at both P100 and P120. Mean values at P140 for all pedometers fluctuated within $\pm 1 \%$ except the TNT model, which underestimated numbers of steps by $\pm 2.8 \%$. All pedometers tended to overestimate and underestimate the numbers of steps at P100 and P140, respectively. Thus, the pedometers were the most accurate at a walking speed of P120

\section{Accuracy of Energy Expenditure Measurements}

All pedometers measured energy expenditure less accurately $(F=20.8, p<0.05)$. Fig. (2) shows the ratios $(\%)$ of pedometer values based on actual energy expenditure as a function of each walking speed. Table 3 shows the statistical outcomes determined from actual energy expenditure as follows: P100: $\mathrm{AC}, 80.7 \%(\mathrm{P}<0.001)$; OM, 53.4\% (P< 0.001); YAMX, 77.3\% (P < 0.001); TNT, 82.6\% (P< 0.001); CTZ, 96.6\%; P120: AC, 80.2\% (P<0.001); OM, 




Fig. (1). Ratios (\%) of pedometer values based on actual number of steps at each walking speed.

Table 2. Statistical Outcomes of Actual Steps While Walking at Different Speeds Determined by Five Japanese Pedometers

\begin{tabular}{|c|c|c|c|c|c|}
\hline Speed & AC & OM & YAMX & TNT & CTZ \\
\hline \hline P100 & $*$ & $* *$ & $N S$ & $N S$ & $* * *$ \\
\hline P120 & $N S$ & $N S$ & $N S$ & $N S$ & $*$ \\
\hline P140 & $N S$ & $N S$ & $N S$ \\
\hline
\end{tabular}

Significance determined using paired t-tests. NS, not significant; * $p<0.05 ; * * p<0.01 ; * * *<<0.001$.

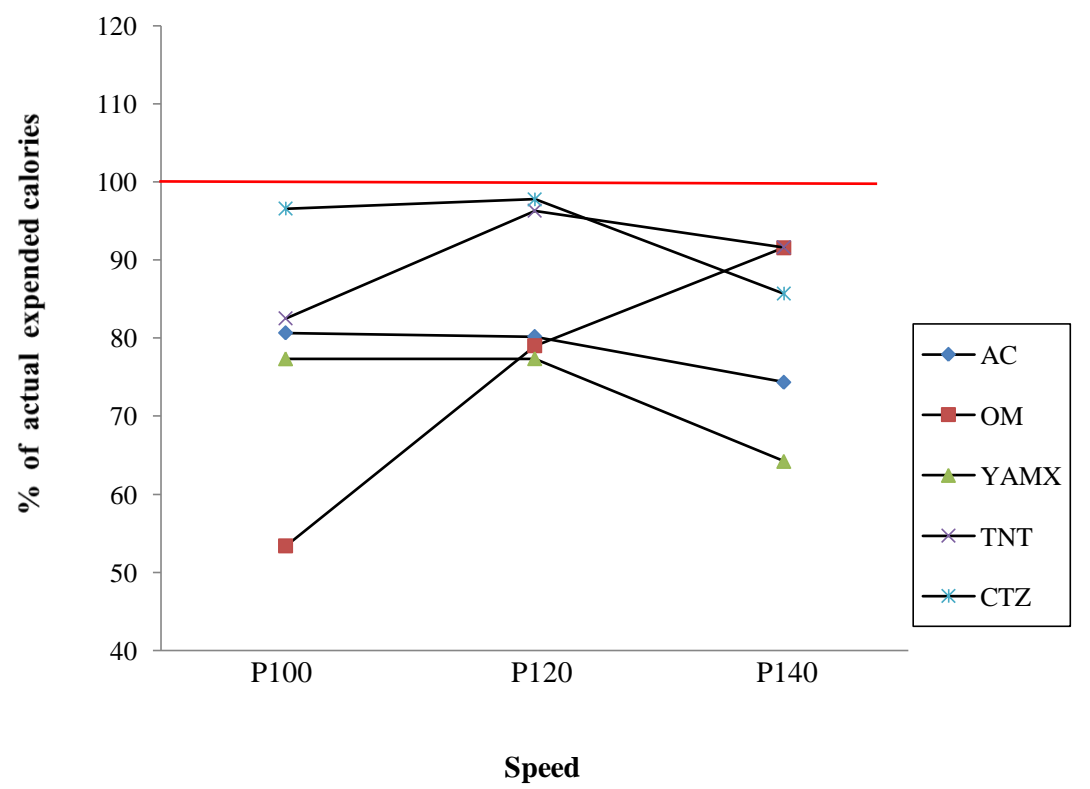

Fig. (2). Ratios (\%) of pedometer values based on actual kilocalories as a function of walking speed. 
Table 3. Statistical Outcomes of Energy Expenditure While Walking at Different Speeds Determined by Five Japanese Pedometers

\begin{tabular}{|c|c|c|c|c|c|}
\hline Speed & AC & OM & YAMX & TNT & CTZ \\
\hline \hline $\mathrm{P} 100$ & $* * *$ & $* * *$ & $* * *$ & NS & NS \\
\hline $\mathrm{P} 120$ & $* * *$ & $* * *$ & $* * *$ & $* *$ & $* *$ \\
\hline $\mathrm{P} 140$ & $* * *$ & $* *$ & $* *$ \\
\hline
\end{tabular}

Significance determined using paired t-tests. NS, not significant; *p<0.05;**p<0.01;***p<0.001.

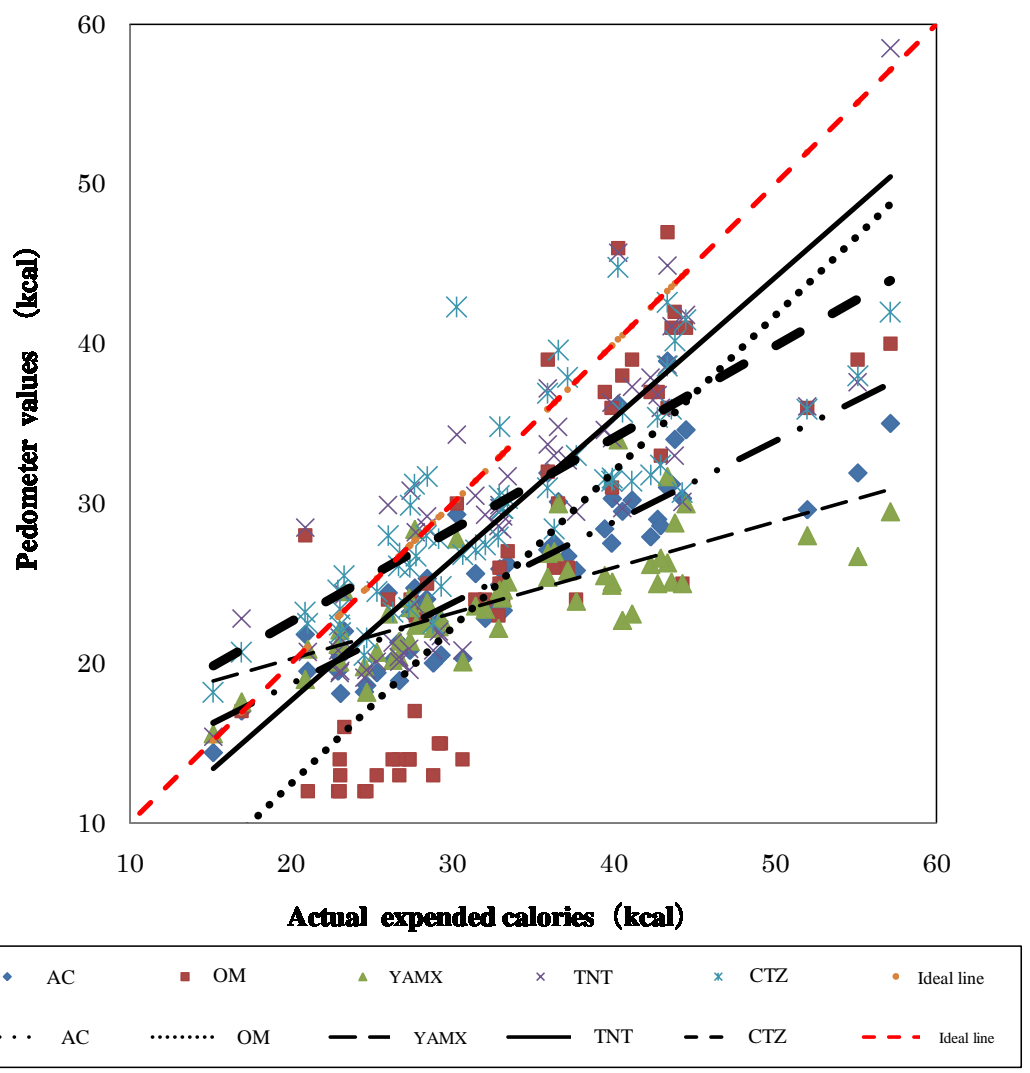

Fig. (3). Regression lines for actual energy expenditure and values generated by pedometers from five manufacturers.

Table 4. Slope and Intercept Relative to Regression (R) Determined from Five Japanese Pedometers

\begin{tabular}{|c|c|c|c|}
\hline Company & Slope & Intercept & \multicolumn{1}{c|}{0.87} \\
\hline \hline AC & 0.50 & 8.56 & 0.84 \\
\hline OM & 0.98 & -7.11 & 0.74 \\
\hline YAMX & 0.29 & 14.57 & 0.84 \\
\hline TNT & 0.77 & 3.94 & 11.11 \\
\hline CTZ & 0.57 & 0.81 \\
\hline
\end{tabular}

$79.0 \%(\mathrm{P}<0.001)$; YAMX, 77.4\% $(\mathrm{P}<0.001)$; TNT, 96.3\%; CTZ, 97.8\%; P140, AC, 74.3\% (P<0.001); OM, 91.6\% (P<0.01); YAMX, 64.2\% (P<0.001); TNT, 91.6\% $(\mathrm{P}<0.01)$; CTZ, 85.7\% $(\mathrm{P}<0.001)$. All pedometers underestimated energy expenditure at all walking speeds. The difference between actual energy expenditure and pedometer values was within $\pm 50 \%$. The average values of all the pedometers were about $80 \%$ at each speed.

Fig. (3) shows scatter plots for actual energy expenditure and values determined by the pedometers from the five manufacturers. The dashed line shows where actual energy expenditure and pedometer values match. Table $\mathbf{4}$ displays 
the slope, intercept and the correlation coefficient estimated from the five regression lines. The correlation coefficients between actual energy expenditure and pedometer values were: AC, 0.87; OM, 0.84; YAMX, 0.74; TNT, 0.84 and CTZ, 0.81 (range, 0.74-0.87).

\section{DISCUSSION}

Fig. (1) shows that the mean values of all five pedometers varied within $\pm 2 \%$ at all speeds, except for that manufactured by TNT, which was $2.8 \%$ for steps measured at P140 $(105 \mathrm{~m} / \mathrm{min})$. Crouter et al. [18] reported that the mean numbers of steps generated by six of 10 pedometers fluctuated within $\pm 1 \%$ of the actual numbers of steps at 80 , 94 and $107 \mathrm{~m} / \mathrm{min}$, and five of these were manufactured in Japan. Schneider et al. [25] determined the accuracy and reliability of 10 pedometers for measuring steps over a 400 $\mathrm{m}$ distance. They found that the Japanese pedometers were the most accurate, being within $\pm 3 \%$ of the actual steps. Our findings are consistent with these results since the mean values of the five Japanese pedometers assessed herein were within $\pm 3 \%$ of the actual values. Notably, the Japanese Industrial Standards (JIS) has set the maximum ratio (\%) of miscounted steps at 3\% [5]. The findings of Crouter and Schneider together with the present findings suggest that Japanese pedometers accurately count steps at all tested speeds, as their step counts are within $1 \%$ and $3 \%$ of the actual values in accordance with the JIS.

All pedometers tended to underestimate actual energy expenditure at P100 $(75 \mathrm{~m} / \mathrm{min}), \mathrm{P} 120(90 \mathrm{~m} / \mathrm{min})$ and P140 (105 m/min) Similarly, Giannakidoku et al. [16] reported that the average values for energy expenditure generated by two Omron pedometers were $28 \%$ lower than the actual values. However, other Omron models tend to overestimate energy expenditure according to one study [24]. Crouter et al. [18] reported that the average values for expended calories determined by eight pedometers were higher than actual values and questioned whether pedometers measure gross or net kilocalories. Energy expenditure reported by some pedometers is calculated from body weight and distance walked [26], which in turn is determined from step counts and stride length. Therefore, errors in measuring energy expenditure might arise from variations in stride length among steps, since five Japanese pedometers generally count steps accurately. Bassett et al. [26] reported that some pedometers provide distance estimates that are reasonably valid at normal walking speeds, but they overestimate and underestimate distance at slow and fast speeds, respectively, due to variations in stride length. Actual stride length varies with walking speed, whereas the stride length entered into a pedometer is constant. This might explain why Japanese pedometers underestimate or overestimate actual energy expenditure.

We examined this issue in more detail by plotting regression lines for actual energy expenditure on the horizontal axis and pedometer values on the vertical axis. Ideal slope and intercept values are close to 1.00 and 0.00 , respectively. A comprehensive evaluation of slope and intercept values produces a correlation coefficient, which when close to 1.00 indicates that values for energy expenditure are accurate. We found that the correlation coefficient between actual and pedometer-determined energy expenditure and was between 0.74 and 0.87 , suggesting that the AC model was the most accurate (correlation coefficient, $0.87)$.

\section{CONCLUSION}

The accuracy of step counts determined using five Japanese pedometers seems quite high because mean values varied within only $1 \%$ and $3 \%$ of actual values. However, five Japanese pedometers tended to underestimate energy expenditure. Several studies have suggested that pedometers are count steps most accurately, calculate distance less accurately and estimate energy expenditure even less accurately [17, 27]. Hatano [28] also reported that the accuracy of counting steps is greater than that for measuring energy expenditure. However, since the average difference between actual and pedometer-generated values for energy expenditure was about $80 \%$ and the correlation coefficient between the two was between 0.74 and 0.87 , five Japanese pedometers reasonably reflect actual energy expenditure, but not as accurately as numbers of steps. It is expected to develop a pedometer that accurately measures energy expenditure in kilocalories in the near future.

\section{CONFLICT OF INTEREST}

The authors confirm that this article content has no conflicts of interest.

\section{ACKNOWLEGEMENTS}

This study was supported by the grant of Preventive Medical Center of Shinshu University Hospital funded by the Japanese Ministry of Education, Culture, Science and Technology. No financial support was received from any of the pedometer companies, importers, or retailers.

\section{REFERENCES}

[1] Kickbusch I. Health promotion, a global perspective. Can J Public Health 1986; 77: 321-7.

[2] Ministry of Health, Labour and Welfare. Available from: http://www.mhlw.go.jp/english/

[3] The American College of Sports Medicine. ACSM's Guideline for exercise testing and prescription, $6^{\text {th }} \mathrm{Ed}$, Philadelphia: Lippincott Williams \& Wilkins 2000.

[4] Pate RR, Pratt M, Blair SN, et al. Physical activity and public health. A recommendation from the Center for Disease Control and Prevention and the American College of Sports Medicine 1995; 273: 402-7.

[5] Hatano Y. Use of the pedometer for promoting daily walking exercise. Int Council Health Phys Educ Recreation J 1993; 29: 4-8.

[6] Paffenbarger RS, Jr, Wing AL, Hyde RT. Physical activity as an index of heart attack risk in college alumni. Am J Epidemiol 1978; 108: $161-75$.

[7] Moreau KL, Degarmo R, Langley J, et al. Increasing daily walking lowers blood pressure in postmenopausal women with borderline to stage I hypertension. Med Sci Sports Exerc 2001; 33: 1825-31.

[8] Swartz AM, Strath SJ, Bassett DR, et al. Increasing daily walking improves glucose tolerance in overweight women. Prev Med 2003; 37: 356-62.

[9] Miller T, Balady G, Fletcher G. Exercise and its role in the prevention and rehabilitation of cardiovascular disease. Ann Behav Med 1997; 19: 220-9.

[10] Iwane M, Arita M, Tomimoto S. Walking 10,000 steps/days or more reduces blood pressure and sympathetic nerve activity in mild essential. Hypertension Res 2000; 23: 573-80.

[11] Boul N, Haddad E, Kenny G, Well G, Sigal R. Effects of exercise on glycemic control and body mass in type 2 diabetes mellitus: a 
meta-analysis of controlled clinical trials. JAMA 2000; 286: 121827.

[12] Stich V, Hainer V, Barbes P, De GI, Parizkora J, Cramper F. Physical activity in the prevention and management of obesity. World Rev Nutr Diet 1997; 82: 219-28.

[13] Fitzpatric SE, Reddy S, Lommel TS, et al. Physical activity and physical function improved following a community-based intervention in older adults in Georgia senior center. J Nutr Elder 2008; 27(1-2): 135-54.

[14] Petersen CB, Severin M, Hancen AW, Curtis T, GrØnbæk M, Tolstrup JS. A population-based randomized controlled trial of the effect of combining a pedometer with an intervention toolkit on physical activity among individuals with low levels of physical activity or fitness. Prev Med 2012; 54(2): 125-30.

[15] Pelssers J, Delecluse C, Opdenacker J, Kennis E, Van Roie E, Boen F. 'Every step counts!'-Effects of structured walking intervention in a community- based senior organization on physical activity, fitness and well-being. J Aging Phys Act 2013; 21: 167-85.

[16] Giannakidou DM, Kambas A, Ageloussis N, et al. The validity of two Omron pedometers during treadmill walking is speed dependent. Eur J Appl Physiol 2012; 112: 49-57.

[17] Basset Jr. DR, Ainsworth BE, Leggett SR, et al. Accuracy of five electronic pedometers for measuring distance walked. Med Sci Sports Exerc 1996; 28: 1071-7.

[18] Crouter SE, Schneider PL, Karabulut M, Basset DR, Jr. Validity of 10 electronic pedometers for measuring steps, distance and energy cost. Med Sci Sports Exerc 2003; 8: 1455-60.

[19] Crouter SE, Schneider PL, Basset DR, Jr. Spring-levered versus piezo-electric pedometer accuracy in overweight and obese adults. Med Sci Sports Exerc 2005; 37(11): 1673-79.
[20] Karabulut M, Crouter SE, Bassett DR, Jr. Comparison of two waist mounted and two ankle mounted electronic pedometers. Eur J Appl Physiol 2005; 95: 335-43.

[21] Le Masurier GC, Tudor-Locke C. Comparison of pedometer and accelerometer accuracy under controlled conditions. Med Sci Sports Exerc 2003; 35(5): 867-71.

[22] Le Masurier G, Lee SM, Tudor-Locke C. Motion sensor accuracy under controlled and free-living conditions. Med Sci Sports Exerc 2004; 36(5): 905-10.

[23] Melanson EL, Knoll JR, Bell ML, Donahoo WT, Hill JO, Nysse LJ, Lanningham-Foster L, Peters JC, Levine JA. Commercially available pedometers: considerations for accurate step counting. Prev Med 2004; 39: 361-68.

[24] Schneider PL, Crouter SE, Lukajic O, Basset DR, Jr. Accuracy and reliability of 10 pedometers for measuring steps over a $400-\mathrm{m}$ walk. Med Sci Sports Exerc 2003; 10: 1779-84.

[25] Swartz AM, Strath SJ, Miller NE, Grimm EK, Ewalt LA, Loy MS, Gennuso KP. Validity of physical activity monitors in assessing energy expenditure in normal, overweight, and obese adults. Open Sports Sci J 2009; 2: 58-64.

[26] Bassett DR, Jr, Mahar MT, Rowe DA, Morrow JR, Jr. Walkingand measurement. Med Sci Sports Exerc 2008; 40(7S): S529-S536.

[27] Bassett DR, Strath SJ. Use of pedometers to assess physical activity. Physical Activity Assessments for Health-Related Research, In: G J Welk Ed. Champaign, IL: Human Kinetics 2002; 166-70.

[28] Hatano Y. Prevelance and use of pedometer. Res J Walk 1997; 1: 45-54.

(C) Osawa et al.; Licensee Bentham Open.

This is an open access article licensed under the terms of the Creative Commons Attribution Non-Commercial License (http://creativecommons.org/licenses/ by-nc/3.0/) which permits unrestricted, non-commercial use, distribution and reproduction in any medium, provided the work is properly cited. 